\title{
Multiple Positive Solutions for a Class of Concave-Convex Semilinear Elliptic Equations in Unbounded Domains with Sign-Changing Weights
}

\author{
Tsing-San Hsu \\ Center for General Education, Chang Gung University, Kwei-Shan, Tao-Yuan 333, Taiwan \\ Correspondence should be addressed to Tsing-San Hsu, tshsu@mail.cgu.edu.tw \\ Received 8 September 2010; Accepted 18 October 2010 \\ Academic Editor: Julio Rossi
}

Copyright (C) 2010 Tsing-San Hsu. This is an open access article distributed under the Creative Commons Attribution License, which permits unrestricted use, distribution, and reproduction in any medium, provided the original work is properly cited.

We study the existence and multiplicity of positive solutions for the following Dirichlet equations: $-\Delta u+u=\lambda a(x)|u|^{q-2} u+b(x)|u|^{p-2} u$ in $\Omega, u=0$ on $\partial \Omega$, where $\lambda>0,1<q<2<p<2^{*}\left(2^{*}=\right.$ $2 N /(N-2)$ if $N \geq 3 ; 2^{*}=\infty$ if $\left.N=1,2\right), \Omega$ is a smooth unbounded domain in $\mathbb{R}^{N}, a(x), b(x)$ satisfy suitable conditions, and $a(x)$ maybe change sign in $\Omega$.

\section{Introduction and Main Results}

In this paper, we deal with the existence and multiplicity of positive solutions for the following semilinear elliptic equation:

$$
\begin{gathered}
-\Delta u+u=\lambda a(x)|u|^{q-2} u+b(x)|u|^{p-2} u \quad \text { in } \Omega, \\
u>0 \quad \text { in } \Omega, \\
u=0 \quad \text { on } \partial \Omega,
\end{gathered}
$$

where $\lambda>0,1<q<2<p<2^{*}\left(2^{*}=2 N /(N-2)\right.$ if $N \geq 3,2^{*}=\infty$ if $\left.N=1,2\right), \Omega \subset \mathbb{R}^{N}$ is an unbounded domain, and $a, b$ are measurable functions and satisfy the following conditions:
(A1) $a \in C(\Omega) \cap L^{q^{*}}(\Omega)\left(q^{*}=p /(p-q)\right)$ with $a^{+}=\max \{a, 0\} \not \equiv 0$ in $\Omega$.
(B1) $b \in C(\Omega) \cap L^{\infty}(\Omega)$ and $b^{+}=\max \{b, 0\} \not \equiv 0$ in $\Omega$. 
Semilinear elliptic equations with concave-convex nonlinearities in bounded domains are widely studied. For example, Ambrosetti et al. [1] considered the following equation:

$$
\begin{gathered}
-\Delta u=\lambda u^{q-1}+u^{p-1} \quad \text { in } \Omega, \\
u>0 \text { in } \Omega, \\
u=0 \text { on } \partial \Omega,
\end{gathered}
$$

where $\lambda>0,1<q<2<p<2^{*}$. They proved that there exists $\lambda_{0}>0$ such that $\left(E_{\curlywedge}\right)$ admits at least two positive solutions for all $\lambda \in\left(0, \lambda_{0}\right)$ and has one positive solution for $\lambda=\lambda_{0}$ and no positive solution for $\lambda>\lambda_{0}$. Actually, Adimurthi et al. [2], Damascelli et al. [3], Ouyang and Shi [4], and Tang [5] proved that there exists $\lambda_{0}>0$ such that $\left(E_{\lambda}\right)$ in the unit ball $B^{N}(0 ; 1)$ has exactly two positive solutions for $\lambda \in\left(0, \lambda_{0}\right)$ and has exactly one positive solution for $\lambda=\lambda_{0}$ and no positive solution exists for $\lambda>\lambda_{0}$. For more general results of $\left(E_{\lambda}\right)$ (involving sign-changing weights) in bounded domains, see Ambrosetti et al. [6], Garcia Azorero et al. [7], Brown and $\mathrm{Wu}$ [8], Brown and Zhang [9], Cao and Zhong [10], de Figueiredo et al. [11], and their references. However, little has been done for this type of problem in unbounded domains. For $\Omega=\mathbb{R}^{N}$, we are only aware of the works [12-15] which studied the existence of solutions for some related concave-convex elliptic problems (not involving sign-changing weights).

Wu in [16] has studied the multiplicity of positive solutions for the following equation involving sign-changing weights:

$$
\begin{gathered}
-\Delta u+u=f_{\lambda}(x) u^{q-1}+g_{\mu}(x) u^{p-1} \quad \text { in } \mathbb{R}^{N}, \\
u>0 \quad \text { in } \mathbb{R}^{N}, \\
u \in H^{1}\left(\mathbb{R}^{N}\right),
\end{gathered}
$$

where $1<q<2<p<2^{*}$, the parameters $\lambda, \mu \geq 0$. He also assumed that $f_{\lambda}(x)=\lambda f_{+}(x)+f_{-}(x)$ is sign-changing and $g_{\mu}(x)=a(x)+\mu b(x)$, where $a$ and $b$ satisfy suitable conditions, and proved $\left(E_{f_{1}, g_{\mu}}\right)$ has at least four positive solutions.

When $\Omega=\Omega^{\prime} \times \mathbb{R}\left(\Omega^{\prime} \subset \mathbb{R}^{N-1}, N \geq 2\right)$ is an infinite strip domain, Wu in [17] considered $\left(E_{\curlywedge a, b}\right)$ (not involving sign-changing weights) assuming that $0 \leq a \in L^{2 /(2-q)}(\Omega), 0 \leq b \in$ $C(\Omega)$ satisfies $\lim _{\left|x_{N}\right| \rightarrow \infty} b\left(x^{\prime}, x_{N}\right)=1$ in $\Omega$ and there exist $\delta>0$ and $0<C_{0}<1$ such that $b\left(x^{\prime}, x_{N}\right) \geq 1-C_{0} e^{-2 \sqrt{1+\theta_{1}+\delta}\left|x_{N}\right|}$ for all $x=\left(x^{\prime}, x_{N}\right) \in \Omega$, where $\theta_{1}$ is the first eigenvalue of the Dirichlet problem $-\Delta$ in $\Omega^{\prime}$. The author proved that there exists a positive constant $\Lambda_{0}$ such that for $\lambda \in\left(0, \Lambda_{0}\right),\left(E_{\mathcal{\lambda} a, b}\right)$ possesses at least two positive solutions.

Miotto and Miyagaki in [18] have studied $\left(E_{\lambda a, b}\right)$ in $\Omega=\Omega^{\prime} \times \mathbb{R}$, under the assumption that $a \in L^{\gamma /(\gamma-q)}(\Omega)\left(q<\gamma \leq 2^{*}\right)$ with $a^{+} \not \equiv 0$ and $a^{-}$is bounded and has a compact support in $\Omega$ and $0 \leq b \in L^{\infty}(\Omega)$ satisfies $\lim _{\left|x_{N}\right| \rightarrow \infty} b\left(x^{\prime}, x_{N}\right)=1$ and there exists $C_{0}>0$ such that $b\left(x^{\prime}, x_{N}\right) \geq 1-C_{0} e^{-2 \sqrt{1+\theta_{1}}\left|x_{N}\right|}$ for all $x=\left(x^{\prime}, x_{N}\right) \in \Omega$, where $\theta_{1}$ is the first eigenvalue of the Dirichlet problem $-\Delta$ in $\Omega^{\prime}$. It was obtained there existence of $\Lambda_{0}>0$ such that for $\lambda \in\left(0, \Lambda_{0}\right)$, $\left(E_{\lfloor a, b}\right)$ possesses at least two positive solutions.

In a recent work [19], Hsu and Lin have studied $\left(E_{\lfloor a, b}\right)$ in $\mathbb{R}^{N}$ under the assumptions (A1)-(A2), (B1), and $\left(\Omega_{b}\right)$. They proved that there exists a constant $\Lambda_{0}>0$ such that for 
$\lambda \in\left(0,(q / 2) \Lambda_{0}\right),\left(E_{\lambda a, b}\right)$ possesses at least two positive solutions. The main aim of this paper is to study $\left(E_{\lambda a, b}\right)$ on the general unbounded domains (see the condition $\left(\Omega_{b}\right)$ ) and extend the results of [19] to more general unbounded domains. We will apply arguments similar to those used in [20] and prove the existence and multiplicity of positive solutions by using Ekeland's variational principle [21].

Set

$$
\Lambda_{0}=\left(\frac{2-q}{(p-q)\left\|b^{+}\right\|_{L^{\infty}}}\right)^{(2-q) /(p-2)}\left(\frac{p-2}{(p-q)\left\|a^{+}\right\|_{L^{q^{*}}}}\right) S_{p}(\Omega)^{(p(2-q) / 2(p-2))+q / 2}>0,
$$

where $\left\|b^{+}\right\|_{L^{\infty}}=\sup _{x \in \Omega} b^{+}(x),\left\|a^{+}\right\|_{L^{*}}=\left(\int_{\Omega}\left|a^{+}\right| q^{*} d x\right)^{1 / q^{*}}$, and $S_{p}(\Omega)$ is the best Sobolev constant for the imbedding of $H_{0}^{1}(\Omega)$ into $L^{p}(\Omega)$. Now, we state the first main result about the existence of positive solution of $\left(E_{\curlywedge a, b}\right)$.

Theorem 1.1. Assume that (A1) and (B1) hold. If $\lambda \in\left(0, \Lambda_{0}\right)$, then $\left(E_{\lambda a, b}\right)$ admits at least one positive solution.

Associated with $\left(E_{\curlywedge a, b}\right)$, we consider the energy functional $J_{\curlywedge a, b}$ in $H_{0}^{1}(\Omega)$ :

$$
J_{\lambda a, b}(u)=\frac{1}{2}\|u\|_{H^{1}}^{2}-\frac{\lambda}{q} \int_{\Omega} a(x)|u|^{q} d x-\frac{1}{p} \int_{\Omega} b(x)|u|^{p} d x
$$

where $\|u\|_{H^{1}}=\left(\int_{\Omega}\left(\left.\nabla u\right|^{2}+u^{2}\right) d x\right)^{1 / 2}$. By Rabinowitz [22, Proposition B.10], J Ja,b $\in$ $C^{1}\left(H_{0}^{1}(\Omega), \mathbb{R}\right)$. It is well known that the solutions of $\left(E_{\lambda a, b}\right)$ are the critical points of the energy functional $J_{\lambda a, b}$ in $H_{0}^{1}(\Omega)$.

Under the assumptions (A1), (B1), and $\lambda>0,\left(E_{\lambda a, b}\right)$ can be regarded as a perturbation problem of the following semilinear elliptic equation:

$$
\begin{gathered}
-\Delta u+u=b(x) u^{p-1} \quad \text { in } \Omega, \\
u>0 \quad \text { in } \Omega, \\
u=0 \quad \text { on } \partial \Omega,
\end{gathered}
$$

where $b(x) \in C(\Omega) \cap L^{\infty}(\Omega)$ and $b(x)>0$ for all $x \in \Omega$. We denote by $S_{p}^{b}(\Omega)$ the best constant which is given by

$$
S_{p}^{b}(\Omega)=\inf _{u \in H_{0}^{1}(\Omega) \backslash\{0\}} \frac{\|u\|_{H^{1}}^{2}}{\left(\int_{\Omega} b(x)|u|^{p} d x\right)^{2 / p}} .
$$


A typical approach for solving problem of this kind is to use the following Minimax method:

$$
\alpha_{\Gamma}^{b}(\Omega)=\inf _{\gamma \in \Gamma(\Omega)} \max _{t \in[0,1]} J_{0}^{b}(\gamma(t))
$$

where

$$
\Gamma(\Omega)=\left\{r \in C\left([0,1], H_{0}^{1}(\Omega)\right): \gamma(0)=0, \gamma(1)=e\right\}
$$

$J_{0}^{b}(e)=0$ and $e \neq 0$. By the Mountain Pass Lemma due to Ambrosetti and Rabinowitz [23], we called the nonzero critical point $u \in H_{0}^{1}(\Omega)$ of $J_{0}^{b}$ a ground state solution of $\left(E_{b}\right)$ in $\Omega$ if $J_{0}^{b}(u)=\alpha_{\Gamma}^{b}(\Omega)$. We remark that the ground state solutions of $\left(E_{b}\right)$ in $\Omega$ can also be obtained by the Nehari minimization problem

$$
\alpha_{0}^{b}(\Omega)=\inf _{v \in \mathcal{M}_{0}^{b}(\Omega)} J_{0}^{b}(v)
$$

where $\mathcal{M}_{0}^{b}(\Omega)=\left\{u \in H_{0}^{1}(\Omega) \backslash\{0\}:\|u\|_{H^{1}}^{2}=\int_{\Omega} b(x)|u|^{p} d x\right\}$. Note that $\mathcal{M}_{0}^{b}(\Omega)$ contains every nonzero solution of $\left(E_{b}\right)$ in $\Omega$,

$$
\alpha_{\Gamma}^{b}(\Omega)=\alpha_{0}^{b}(\Omega)=\frac{p-2}{2 p} S_{p}^{b}(\Omega)^{p /(p-2)}>0
$$

(see Willem [24]), and if $b(x) \equiv b^{\infty}>0$ is a constant, then $J_{0}^{b}$ and $\alpha_{0}^{b}(\Omega)$ replace $J_{0}$ and $\alpha_{0}^{\infty}(\Omega)$, respectively.

The existence of ground state solutions of $\left(E_{b}\right)$ is affected by the shape of the domain $\Omega$ and $b(x)$ that satisfies some suitable conditions and has been the focus of a great deal of research in recent years. By the Rellich compactness theorem and the Minimax method, it is easy to obtain a ground state solution for $\left(E_{b}\right)$ in bounded domains. When $\Omega$ is an unbounded domain and $b(x) \equiv b^{\infty}$, the existence of ground state solutions has been established by several authors under various conditions. We mention, in particular, results by Berestycki and Lions [25], Lien et al. [26], Chen and Wang [27], and Del Pino and Felmer [28, 29]. In [25], $\Omega=\mathbb{R}^{N}$. Actually, Kwong [30] proved that the positive solution of $\left(E_{b}\right)$ in $\mathbb{R}^{N}$ is unique. In [26], for $\Omega$ is a periodic domain. In $[26,27]$, the domain $\Omega$ is required to satisfy

$\left(\Omega_{1}\right) \Omega=\Omega_{1} \cup \Omega_{2}$, where $\Omega_{1}, \Omega_{2}$ are domains in $\mathbb{R}^{N}$ and $\Omega_{1} \cap \Omega_{2}$ is bounded;

$\left(\Omega_{2}\right) \alpha_{0}^{\infty}(\Omega)<\min \left\{\alpha_{0}^{\infty}\left(\Omega_{1}\right), \alpha_{0}^{\infty}\left(\Omega_{2}\right)\right\}$.

In $[28,29]$ for $1 \leq l \leq N-1, \mathbb{R}^{N}=\mathbb{R}^{l} \times \mathbb{R}^{N-l}$. For a point $x \in \mathbb{R}^{N}$, we have $x=(y, z)$, where $y \in \mathbb{R}^{l}$ and $z \in \mathbb{R}^{N-l}$. Let $y \in \mathbb{R}^{l}$, we denote by $\Omega^{y} \subset \mathbb{R}^{N-l}$ the projection of $\Omega$ onto $\mathbb{R}^{N-l}$, that is,

$$
\Omega^{y}=\left\{z \in \mathbb{R}^{N-l}:(y, z) \in \Omega\right\} .
$$


The domain $\Omega$ satisfies the following conditions:

$\left(\Omega_{3}\right) \Omega$ is a smooth subset of $\mathbb{R}^{N}$ and the projections $\Omega^{y}$ are bounded uniformly in $y \in \mathbb{R}^{l}$;

$\left(\Omega_{4}\right)$ there exists a nonempty closed set $D \subset \mathbb{R}^{N-l}$ such that $D \subset \Omega^{y}$ for all $y \in \mathbb{R}^{l}$;

$\left(\Omega_{5}\right)$ for each $\delta>0$, there exists $R_{0}>0$ such that

$$
\Omega^{y} \subset\left\{z \in \mathbb{R}^{N-l}: \operatorname{dist}(z, D)<\delta\right\}
$$

for all $|y| \geq R_{0}$.

When $b(x) \not \equiv b^{\infty}$ and $b(x) \in C(\Omega) \cap L^{\infty}(\Omega)$, the existence of ground state solutions of $\left(E_{b}\right)$ has been established by the condition $b(x) \geq b^{\infty}$ and the existence of ground state solutions of limit equation

$$
\begin{gathered}
-\Delta u+u=b^{\infty} u^{p-1} \quad \text { in } \Omega, \\
u>0 \quad \text { in } \Omega, \\
u=0 \quad \text { on } \partial \Omega .
\end{gathered}
$$

In order to get the second positive solution of $\left(E_{\lambda a, b}\right)$, we need some additional assumptions for $a(x), b(x)$, and $\Omega$. We assume the following conditions on $a(x), b(x)$, and $\Omega$ :

$\left(\Omega_{b}\right) b(x)>0$ for all $x \in \Omega$ and $\left(E_{b}\right)$ in $\Omega$ has a ground state solution $w_{0}$ such that $J_{0}^{b}\left(w_{0}\right)=\alpha_{0}^{b}(\Omega)$.

(A2) $\int_{\Omega} a(x)\left|w_{0}\right|^{q} d x>0$ where $w_{0}$ is a positive ground state solution of $\left(E_{b}\right)$ in $\Omega$.

Theorem 1.2. Assume that (A1)-(A2), (B1), and $\left(\Omega_{b}\right)$ hold. If $\lambda \in\left(0,(q / 2) \Lambda_{0}\right),\left(E_{\lambda a, b}\right)$ admits at least two positive solutions.

Throughout this paper, (A1) and (B1) will be assumed. $H_{0}^{1}(\Omega)$ denotes the standard Sobolev space, whose norm $\|\cdot\|_{H^{1}}$ is induced by the standard inner product. The dual space of $H_{0}^{1}(\Omega)$ will be denoted by $H^{-1}(\Omega) .\langle\cdot, \cdot\rangle$ denote the usual scalar product in $H_{0}^{1}(\Omega)$. We denote the norm in $L^{s}(\Omega)$ by $\|\cdot\|_{L^{s}}$ for $1 \leq s \leq \infty . o_{n}(1)$ denotes $o_{n}(1) \rightarrow 0$ as $n \rightarrow \infty$. C, $C_{i}$ will denote various positive constants, the exact values of which are not important. This paper is organized as follows. In Section 2, we give some properties of Nehari manifold. In Sections 3 and 4, we complete proofs of Theorems 1.1 and 1.2.

\section{Nehari Manifold}

In this section, we will give some properties of Nehari manifold. As the energy functional $J_{\lambda a, b}$ is not bounded below on $H_{0}^{1}(\Omega)$, it is useful to consider the functional on the Nehari manifold

$$
\mathcal{M}_{\lambda a, b}(\Omega)=\left\{u \in H_{0}^{1}(\Omega) \backslash\{0\}:\left\langle\left(J_{\curlywedge a, b}\right)^{\prime}(u), u\right\rangle=0\right\} .
$$


Thus, $u \in \mathcal{M}_{\lambda a, b}(\Omega)$ if and only if

$$
\left\langle\left(J_{\lambda a, b}\right)^{\prime}(u), u\right\rangle=\|u\|_{H^{1}}^{2}-\lambda \int_{\Omega} a(x)|u|^{q} d x-\int_{\Omega} b(x)|u|^{p} d x=0 .
$$

Note that $\mathcal{M}_{\lambda a, b}(\Omega)$ contains every nonzero solution of $\left(E_{\lambda a, b}\right)$. Moreover, we have the following results.

Lemma 2.1. The energy functional $J_{\lambda a, b}$ is coercive and bounded below on $\mathcal{M}_{\lambda a, b}(\Omega)$.

Proof. If $u \in \mathcal{M}_{\curlywedge a, b}(\Omega)$, then by (A1), (2.2), Hölder and Sobolev inequalities

$$
\begin{aligned}
J_{\lambda a, b}(u) & =\frac{p-2}{2 p}\|u\|_{H^{1}}^{2}-\lambda\left(\frac{p-q}{p q}\right) \int_{\Omega} a(x)|u|^{q} d x \\
& \geq \frac{p-2}{2 p}\|u\|_{H^{1}}^{2}-\lambda\left(\frac{p-q}{p q}\right) S_{p}(\Omega)^{-q / 2}\left\|a^{+}\right\|_{L^{q^{*}}}\|u\|_{H^{1}}^{q}
\end{aligned}
$$

Thus, $J_{\lambda a, b}$ is coercive and bounded below on $\mathcal{M}_{\lambda a, b}(\Omega)$.

Define

$$
\psi_{\lambda a, b}(u)=\left\langle\left(J_{\lambda a, b}\right)^{\prime}(u), u\right\rangle
$$

Then for $u \in \mathcal{M}_{\lambda a, b}(\Omega)$,

$$
\begin{aligned}
\left\langle\left(\psi_{\curlywedge a, b}\right)^{\prime}(u), u\right\rangle & =2\|u\|_{H^{1}}^{2}-\curlywedge q \int_{\Omega} a(x)|u|^{q} d x-p \int_{\Omega} b(x)|u|^{p} d x \\
& =(2-q)\|u\|_{H^{1}}^{2}-(p-q) \int_{\Omega} b(x)|u|^{p} d x \\
& =\lambda(p-q) \int_{\Omega} a(x)|u|^{q} d x-(p-2)\|u\|_{H^{1}}^{2}
\end{aligned}
$$

Similar to the method used in Tarantello [20], we split $\mathcal{M}_{\lambda a, b}(\Omega)$ into three parts:

$$
\begin{aligned}
& \mathcal{M}_{\lambda a, b}^{+}(\Omega)=\left\{u \in \mathcal{M}_{\curlywedge a, b}(\Omega):\left\langle\left(\psi_{\curlywedge a, b}\right)^{\prime}(u), u\right\rangle>0\right\}, \\
& \mathcal{M}_{\curlywedge a, b}^{0}(\Omega)=\left\{u \in \mathcal{M}_{\curlywedge a, b}(\Omega):\left\langle\left(\psi_{\curlywedge a, b}\right)^{\prime}(u), u\right\rangle=0\right\}, \\
& \mathcal{M}_{\curlywedge a, b}^{-}(\Omega)=\left\{u \in \mathcal{M}_{\curlywedge a, b}(\Omega):\left\langle\left(\psi_{\curlywedge a, b}\right)^{\prime}(u), u\right\rangle<0\right\} .
\end{aligned}
$$

Then, we have the following results. 
Lemma 2.2. Assume that $u_{\lambda}$ is a local minimizer for $J_{\lambda a, b}$ on $\mathcal{M}_{\lambda a, b}(\Omega)$ and $u_{\lambda} \notin \mathcal{M}_{\lambda a, b}^{0}(\Omega)$. Then $\left(J_{\curlywedge a, b}\right)^{\prime}\left(u_{\curlywedge}\right)=0$ in $H^{-1}(\Omega)$.

Proof. Our proof is almost the same as that in Brown and Zhang [9, Theorem 2.3] (or see Binding et al. [31]).

Lemma 2.3. We have the following.

(i) If $u \in \mathcal{M}_{\lambda a, b}^{+}(\Omega) \cup \mathcal{M}_{\lambda a, b}^{0}(\Omega)$, then $\int_{\Omega} a(x)|u|^{q} d x>0$;

(ii) If $u \in \mathcal{M}_{\lambda a, b}^{-}(\Omega)$, then $\int_{\Omega} b(x)|u|^{p} d x>0$.

Proof. The proof is immediate from (2.6) and (2.7).

Moreover, we have the following result.

Lemma 2.4. If $\lambda \in\left(0, \Lambda_{0}\right)$, then $\mathcal{M}_{\lambda a, b}^{0}(\Omega)=\emptyset$ where $\Lambda_{0}$ is the same as in (1.1).

Proof. Suppose the contrary. Then there exists $\lambda \in\left(0, \Lambda_{0}\right)$ such that $\mathcal{M}_{\lambda a, b}^{0}(\Omega) \neq \emptyset$. Then for $u \in \mathcal{M}_{\lambda a, b}^{0}(\Omega)$ by (2.6) and Sobolev inequality, we have

$$
\frac{2-q}{p-q}\|u\|_{H^{1}}^{2}=\int_{\Omega} b(x)|u|^{p} d x \leq\left\|b^{+}\right\|_{L^{\infty}} S_{p}(\Omega)^{-p / 2}\|u\|_{H^{1}}^{p}
$$

and so

$$
\|u\|_{H^{1}} \geq\left(\frac{2-q}{(p-q)\left\|b^{+}\right\|_{L^{\infty}}}\right)^{1 /(p-2)} S_{p}(\Omega)^{p / 2(p-2)}
$$

Similarly, using (2.7) and Hölder and Sobolev inequalities, we have

$$
\|u\|_{H^{1}}^{2}=\lambda \frac{p-q}{p-2} \int_{\Omega} a(x)|u|^{q} d x \leq \lambda \frac{p-q}{p-2}\left\|a^{+}\right\|_{L^{q^{*}}} S_{p}(\Omega)^{-q / 2}\|u\|_{H^{1}}^{q}
$$

which implies

$$
\|u\|_{H^{1}} \leq\left(\lambda \frac{p-q}{p-2}\left\|a^{+}\right\|_{L^{q^{*}}}\right)^{1 /(2-q)} S_{p}(\Omega)^{-q / 2(2-q)} .
$$

Hence, we must have

$$
\lambda \geq\left(\frac{2-q}{(p-q)\left\|b^{+}\right\|_{L^{\infty}}}\right)^{(2-q) /(p-2)}\left(\frac{p-2}{(p-q)\left\|a^{+}\right\|_{L^{q^{*}}}}\right) S_{p}(\Omega)^{(p(2-q) / 2(p-2))+q / 2}=\Lambda_{0},
$$

which is a contradiction. This completes the proof. 
For each $u \in H_{0}^{1}(\Omega)$ with $\int_{\Omega} b(x)|u|^{p} d x>0$, we write

$$
t_{\max }(u)=\left(\frac{(2-q)\|u\|_{H^{1}}^{2}}{(p-q) \int_{\Omega} b(x)|u|^{p}} d x\right)^{1 /(p-2)}>0 .
$$

Then the following lemma holds.

Lemma 2.5. Let $\lambda \in\left(0, \Lambda_{0}\right)$. For each $u \in H_{0}^{1}(\Omega)$ with $\int_{\Omega} b(x)|u|^{p} d x>0$, we have the following. (i) If $\int_{\Omega} a(x)|u|^{q} d x \leq 0$, then there is a unique $t^{-}=t^{-}(u)>t_{\max }(u)$ such that $t^{-} u \in \mathcal{M}_{\lambda a, b}^{-}(\Omega)$

$$
J_{\lambda a, b}\left(t^{-} u\right)=\sup _{t \geq 0} J_{\lambda a, b}(t u)
$$

(ii) If $\int_{\Omega} a(x)|u|^{q} d x>0$, then there are unique

$$
0<t^{+}=t^{+}(u)<t_{\max }(u)<t^{-}=t^{-}(u)
$$

such that $t^{+} u \in \mathcal{M}_{\lambda a, b}^{+}(\Omega), t^{-} u \in \mathcal{M}_{\lambda a, b}^{-}(\Omega)$, and

$$
J_{\curlywedge a, b}\left(t^{+} u\right)=\inf _{0 \leq t \leq t_{\max }(u)} J_{\lambda a, b}(t u), \quad J_{\lambda a, b}\left(t^{-} u\right)=\sup _{t \geq 0} J_{\curlywedge a, b}(t u) .
$$

Proof. The proof is almost the same as that in $\mathrm{Wu}[32$, Lemma 5] and is omitted here.

\section{Proof of Theorem 1.1}

First, we remark that it follows Lemma 2.4 that

$$
\mathcal{M}_{\lambda a, b}(\Omega)=\mathcal{M}_{\lambda a, b}^{+}(\Omega) \cup \mathcal{M}_{\lambda a, b}^{-}(\Omega)
$$

for all $\lambda \in\left(0, \Lambda_{0}\right)$. Furthermore, by Lemma 2.5 it follows that $\mathcal{M}_{\lambda a, b}^{+}(\Omega)$ and $\mathcal{M}_{\lambda a, b}^{-}(\Omega)$ are nonempty, and by Lemma 2.1 we may define

$$
\alpha_{\lambda a, b}=\inf _{u \in \mathcal{M}_{\lambda a, b}(\Omega)} J_{\lambda a, b}(u) ; \alpha_{\lambda a, b}^{+}=\inf _{u \in \mathcal{M}_{\lambda a, b}^{+}(\Omega)} J_{\lambda a, b}(u) ; \alpha_{\lambda a, b}^{-}=\inf _{u \in \mathcal{M}_{\lambda a, b}^{-}(\Omega)} J_{\lambda a, b}(u) .
$$

Then we get the following result.

Theorem 3.1. We have the following.

(i) If $\lambda \in\left(0, \Lambda_{0}\right)$, then we have $\alpha_{\lambda a, b}^{+}<0$.

(ii) If $\lambda \in\left(0,(q / 2) \Lambda_{0}\right)$, then $\alpha_{\lambda a, b}^{-}>d_{0}$ for some $d_{0}>0$.

In particular, for each $\lambda \in\left(0,(q / 2) \Lambda_{0}\right)$, we have $\alpha_{\lambda a, b}^{+}=\alpha_{\lambda a, b}$. 
Proof. (i) Let $u \in \mathcal{M}_{\lambda a, b}^{+}(\Omega)$. By (2.6),

$$
\frac{2-q}{p-q}\|u\|_{H^{1}}^{2}>\int_{\Omega} b(x)|u|^{p} d x
$$

and so

$$
\begin{aligned}
J_{\lambda}(u) & =\left(\frac{1}{2}-\frac{1}{q}\right)\|u\|_{H^{1}}^{2}+\left(\frac{1}{q}-\frac{1}{p}\right) \int_{\Omega} b(x)|u|^{p} d x<\left[\left(\frac{1}{2}-\frac{1}{q}\right)+\left(\frac{1}{q}-\frac{1}{p}\right)\left(\frac{2-q}{p-q}\right)\right]\|u\|_{H^{1}}^{2} \\
& =-\frac{(p-2)(2-q)}{2 p q}\|u\|_{H^{1}}^{2}<0 .
\end{aligned}
$$

Therefore, $\alpha_{\lambda a, b}^{+}<0$.

(ii) Let $u \in \mathcal{M}_{\lambda a, b}^{-}(\Omega)$. By (2.6),

$$
\frac{2-q}{p-q}\|u\|_{H^{1}}^{2}<\int_{\Omega} b(x)|u|^{p} d x
$$

Moreover, by (B1) and Sobolev inequality theorem,

$$
\int_{\Omega} b(x)|u|^{p} d x \leq\left\|b^{+}\right\|_{L^{\infty}} S_{p}(\Omega)^{-p / 2}\|u\|_{H^{1}}^{p}
$$

This implies

$$
\|u\|_{H^{1}}>\left(\frac{2-q}{(p-q)\left\|b^{+}\right\|_{L^{\infty}}}\right)^{1 /(p-2)} S_{p}(\Omega)^{p / 2(p-2)} \quad \forall u \in \mathcal{M}_{\lambda a, b}^{-}(\Omega) .
$$

By (2.4) and (3.7), we have

$$
\begin{aligned}
& J_{\lambda a, b}(u) \\
& \quad \geq\|u\|_{H^{1}}^{q}\left[\frac{p-2}{2 p}\|u\|_{H^{1}}^{2-q}-\lambda\left(\frac{p-q}{p q}\right) S_{p}(\Omega)^{-q / 2}\left\|a^{+}\right\|_{L^{*}}\right] \\
& >\left(\frac{2-q}{(p-q)\left\|b^{+}\right\|_{L^{\infty}}}\right)^{q /(p-2)} S_{p}(\Omega)^{p q / 2(p-2)} \\
& \quad \times\left[\frac{p-2}{2 p} S_{p}(\Omega)^{p(2-q) / 2(p-2)}\left(\frac{2-q}{(p-q)\left\|b^{+}\right\|_{L^{\infty}}}\right)^{(2-q) /(p-2)}-\lambda\left(\frac{p-q}{p q}\right) S_{p}(\Omega)^{-q / 2}\left\|a^{+}\right\|_{L^{q^{*}}}\right] .
\end{aligned}
$$


Thus, if $\lambda \in\left(0,(q / 2) \Lambda_{0}\right)$, then

$$
J_{\lambda a, b}(u)>d_{0} \quad \forall u \in \mathcal{M}_{\lambda a, b}^{-}(\Omega),
$$

for some positive constant $d_{0}$. This completes the proof.

We define the Palais-Smale (simply by $(P S)$ ) sequences, $(P S)$-values, and (PS)conditions in $H_{0}^{1}(\Omega)$ for $J_{\lambda a, b}$ as follows.

Definition 3.2. (i) For $c \in \mathbb{R}$, a sequence $\left\{u_{n}\right\}$ is a $(P S)_{c}$-sequence in $H_{0}^{1}(\Omega)$ for $J_{\lambda a, b}$ if $J_{\lambda a, b}\left(u_{n}\right)=$ $c+o_{n}(1)$ and $\left(J_{\lambda a, b}\right)^{\prime}\left(u_{n}\right)=o_{n}(1)$ strongly in $H^{-1}(\Omega)$ as $n \rightarrow \infty$.

(ii) $c \in \mathbb{R}$ is a (PS)-value in $H_{0}^{1}(\Omega)$ for $J_{\lambda a, b}$ if there exists a $(P S)_{c}$-sequence in $H_{0}^{1}(\Omega)$ for $J_{\lambda a, b}$.

(iii) $J_{\lambda a, b}$ satisfies the $(P S)_{c}$-condition in $H_{0}^{1}(\Omega)$ if any $(P S)_{c}$-sequence $\left\{u_{n}\right\}$ in $H_{0}^{1}(\Omega)$ for $J_{\lambda a, b}$ contains a convergent subsequence.

Now, we use the Ekeland variational principle [21] to get the following results.

Proposition 3.3. (i) If $\lambda \in\left(0, \Lambda_{0}\right)$, then there exists a $(P S)_{\alpha_{\lambda a, b}}$-sequence $\left\{u_{n}\right\} \subset \mathcal{M}_{\lambda a, b}(\Omega)$ in $H_{0}^{1}(\Omega)$ for $J_{\lambda a, b}$.

(ii) If $\lambda \in\left(0,(q / 2) \Lambda_{0}\right)$, then there exists a $(P S)_{\alpha_{\lambda a, b}^{-}}$-sequence $\left\{u_{n}\right\} \subset \mathcal{M}_{\lambda a, b}^{-}(\Omega)$ in $H_{0}^{1}(\Omega)$ for $J_{\lambda a, b}$.

Proof. The proof is almost the same as that in $\mathrm{Wu}$ [32, Proposition 9].

Now, we establish the existence of a local minimum for $J_{\lambda a, b}$ on $\mathcal{M}_{\lambda a, b}^{+}(\Omega)$.

Theorem 3.4. Assume (A1) and (B1) hold. If $\lambda \in\left(0, \Lambda_{0}\right)$, then $J_{\lambda a, b}$ has a minimizer $u_{\lambda}$ in $\mathcal{M}_{\lambda a, b}^{+}(\Omega)$ and it satisfies the following.

(i) $J_{\lambda a, b}\left(u_{\lambda}\right)=\alpha_{\lambda a, b}=\alpha_{\lambda a, b}^{+}$.

(ii) $u_{\curlywedge}$ is a positive solution of $\left(E_{\lambda a, b}\right)$ in $\Omega$.

(iii) $\left\|u_{\lambda}\right\|_{H^{1}} \rightarrow 0$ as $\lambda \rightarrow 0^{+}$.

Proof. By Proposition 3.3(i), there is a minimizing sequence $\left\{u_{n}\right\}$ for $J_{\lambda a, b}$ on $\mathcal{M}_{\lambda a, b}(\Omega)$ such that

$$
J_{\lambda a, b}\left(u_{n}\right)=\alpha_{\lambda a, b}+o_{n}(1), \quad\left(J_{\lambda a, b}\right)^{\prime}\left(u_{n}\right)=o_{n}(1) \text { in } H^{-1}(\Omega) .
$$

Since $J_{\lambda}$ is coercive on $\mathcal{M}_{\lambda a, b}(\Omega)$ (see Lemma 2.1), we get that $\left\{u_{n}\right\}$ is bounded in $H_{0}^{1}(\Omega)$. Going if necessary to a subsequence, we can assume that there exists $u_{\lambda} \in H_{0}^{1}(\Omega)$ such that

$$
\begin{aligned}
& u_{n} \rightarrow u_{\lambda} \quad \text { weakly in } H_{0}^{1}(\Omega), \\
& u_{n} \longrightarrow u_{\lambda} \quad \text { almost every where in } \Omega, \\
& u_{n} \longrightarrow u_{\curlywedge} \quad \text { strongly in } L_{\text {loc }}^{s}(\Omega) \forall 1 \leq s<2^{*} .
\end{aligned}
$$

By (A1), Egorov theorem, and Hölder inequality, we have

$$
\lambda \int_{\Omega} a(x)\left|u_{n}\right|^{q} d x=\lambda \int_{\Omega} a(x)\left|u_{\lambda}\right|^{q} d x+o_{n}(1) \quad \text { as } n \longrightarrow \infty
$$


First, we claim that $u_{\lambda}$ is a nonzero solution of $\left(E_{\lambda a, b}\right)$. By (3.10) and (3.11), it is easy to see that $u_{\curlywedge}$ is a solution of $\left(E_{\curlywedge a, b}\right)$. From $u_{n} \in \mathcal{M}_{\curlywedge a, b}(\Omega)$ and (2.3), we deduce that

$$
\lambda \int_{\Omega} a(x)\left|u_{n}\right|^{q} d x=\frac{q(p-2)}{2(p-q)}\left\|u_{n}\right\|_{H^{1}}^{2}-\frac{p q}{p-q} J_{\lambda a, b}\left(u_{n}\right)
$$

Let $n \rightarrow \infty$ in (3.13); by (3.10), (3.12), and $\alpha_{\curlywedge a, b}<0$, we get

$$
\lambda \int_{\Omega} a(x)\left|u_{\curlywedge}\right|^{q} d x \geq-\frac{p q}{p-q} \alpha_{\lambda a, b}>0
$$

Thus, $u_{\lambda} \in \mathcal{M}_{\lambda a, b}(\Omega)$ is a nonzero solution of $\left(E_{\lambda a, b}\right)$. Now we prove that $u_{n} \rightarrow u_{\curlywedge}$ strongly in $H_{0}^{1}(\Omega)$ and $J_{\lambda a, b}\left(u_{\curlywedge}\right)=\alpha_{\lambda a, b}$. By (3.13), if $u \in \mathcal{M}_{\lambda a, b}(\Omega)$, then

$$
J_{\lambda a, b}(u)=\frac{p-2}{2 p}\|u\|_{H^{1}}^{2}-\frac{p-q}{p q} \lambda \int_{\Omega} a(x)|u|^{q} d x
$$

In order to prove that $J_{\lambda a, b}\left(u_{\curlywedge}\right)=\alpha_{\lambda a, b}$, it suffices to recall that $u_{n}, u_{\curlywedge} \in \mathcal{M}_{\lambda a, b}(\Omega)$, by (3.15) and by applying Fatou's lemma to get

$$
\begin{aligned}
\alpha_{\lambda a, b} & \leq J_{\lambda a, b}\left(u_{\curlywedge}\right)=\frac{p-2}{2 p}\left\|u_{\curlywedge}\right\|_{H^{1}}^{2}-\frac{p-q}{p q} \lambda \int_{\Omega} a(x)\left|u_{\curlywedge}\right|^{q} d x \\
& \leq \liminf _{n \rightarrow \infty}\left(\frac{p-2}{2 p}\left\|u_{n}\right\|_{H^{1}}^{2}-\frac{p-q}{p q} \lambda \int_{\Omega} a(x)\left|u_{n}\right|^{q} d x\right) \\
& \leq \liminf _{n \rightarrow \infty} J_{\lambda a, b}\left(u_{n}\right)=\alpha_{\lambda a, b} .
\end{aligned}
$$

This implies that $J_{\lambda a, b}\left(u_{\curlywedge}\right)=\alpha_{\lambda a, b}$ and $\lim _{n \rightarrow \infty}\left\|u_{n}\right\|_{H^{1}}^{2}=\left\|u_{\curlywedge}\right\|_{H^{1}}^{2}$. Let $v_{n}=u_{n}-u_{\curlywedge}$; then by Brézis and Lieb, lemma [33] implies that

$$
\left\|u_{n}\right\|_{H^{1}}^{2}=\left\|u_{n}\right\|_{H^{1}}^{2}-\left\|u_{\lambda}\right\|_{H^{1}}^{2}+o_{n}(1)
$$

Therefore, $u_{n} \rightarrow u_{\curlywedge}$ strongly in $H_{0}^{1}(\Omega)$. Moreover, we have $u_{\lambda} \in \mathcal{M}_{\lambda a, b}^{+}(\Omega)$. On the contrary, if $u_{\lambda} \in \mathcal{M}_{\lambda a, b}^{-}(\Omega)$, then by Lemma 2.5 , there are unique $t_{0}^{+}$and $t_{0}^{-}$such that $t_{0}^{+} u_{\lambda} \in \mathcal{M}_{\lambda a, b}^{+}(\Omega)$ and $t_{0}^{-} u_{\lambda} \in \mathcal{M}_{\lambda a, b}^{-}(\Omega)$. In particular, we have $t_{0}^{+}<t_{0}^{-}=1$. Since

$$
\frac{d}{d t} J_{\lambda a, b}\left(t_{0}^{+} u_{\curlywedge}\right)=0, \quad \frac{d^{2}}{d t^{2}} J_{\lambda a, b}\left(t_{0}^{+} u_{\curlywedge}\right)>0
$$


there exists $t_{0}^{+}<\bar{t} \leq t_{0}^{-}$such that $J_{\curlywedge a, b}\left(t_{0}^{+} u_{\curlywedge}\right)<J_{\curlywedge a, b}\left(\bar{t} u_{\curlywedge}\right)$. By Lemma 2.5,

$$
J_{\lambda a, b}\left(t_{0}^{+} u_{\curlywedge}\right)<J_{\lambda a, b}\left(\bar{t} u_{\lambda}\right) \leq J_{\lambda a, b}\left(t_{0}^{-} u_{\curlywedge}\right)=J_{\lambda a, b}\left(u_{\curlywedge}\right)
$$

which is a contradiction. Since $J_{\lambda a, b}\left(u_{\lambda}\right)=J_{\lambda a, b}\left(\left|u_{\lambda}\right|\right)$ and $\left|u_{\lambda}\right| \in \mathcal{M}_{\lambda a, b}^{+}(\Omega)$, by Lemma 2.2 we may assume that $u_{\curlywedge}$ is a nonzero nonnegative solution of $\left(E_{\curlywedge a, b}\right)$. By Harnack inequality [34], we deduce that $u_{\curlywedge}>0$ in $\Omega$. Finally, by (2.3) and Hölder and Sobolev inequalities,

$$
\left\|u_{\curlywedge}\right\|_{H^{1}}^{2-q}<\lambda \frac{p-q}{p-2}\left\|a^{+}\right\|_{L^{q^{*}}} S_{p}(\Omega)^{-q / 2}
$$

and so $\left\|u_{\curlywedge}\right\|_{H^{1}} \rightarrow 0$ as $\lambda \rightarrow 0^{+}$.

Now, we begin the proof of Theorem 1.1. By Theorem 3.4, we obtain that $\left(E_{\lambda a, b}\right)$ has a positive solution $u_{\lambda}$ in $H_{0}^{1}(\Omega)$.

\section{Proof of Theorem 1.2}

In this section, we will establish the existence of the second positive solution of $\left(E_{\curlywedge a, b}\right)$ by proving that $J_{\lambda a, b}$ satisfies the $(P S)_{\alpha_{\lambda a, b}^{-}}$-condition.

Lemma 4.1. Assume that (A1) and (B1) hold. If $\left\{u_{n}\right\} \subset H_{0}^{1}(\Omega)$ is a (PS) $)_{c}$-sequence for $J_{\lambda a, b}$, then $\left\{u_{n}\right\}$ is bounded in $H_{0}^{1}(\Omega)$.

Proof. We argue by contradiction. Assume that $\left\|u_{n}\right\|_{H^{1}} \rightarrow \infty$. Let $\widehat{u}_{n}=u_{n} /\left\|u_{n}\right\|_{H^{1}}$. We may assume that $\widehat{u}_{n} \rightarrow \widehat{u}$ weakly in $H_{0}^{1}(\Omega)$. This implies that $\widehat{u}_{n} \rightarrow \widehat{u}$ strongly in $L_{\text {loc }}^{s}(\Omega)$ for all $1 \leq s<2^{*}$. By (A1), Egorov theorem, and Hölder inequality, we have

$$
\frac{\lambda}{q} \int_{\Omega} a(x)\left|\widehat{u}_{n}\right|^{q} d x=\frac{\lambda}{q} \int_{\Omega} a(x)|\widehat{u}|^{q} d x+o_{n}(1)
$$

Since $\left\{u_{n}\right\}$ is a $(P S)_{c}$-sequence for $J_{\lambda a, b}$ and $\left\|u_{n}\right\|_{H^{1}} \rightarrow \infty$, there hold

$$
\begin{gathered}
\frac{1}{2}\left\|\widehat{u}_{n}\right\|_{H^{1}}^{2}-\frac{\lambda\left\|u_{n}\right\|_{H^{1}}^{q-2}}{q} \int_{\Omega} a(x)\left|\widehat{u}_{n}\right|^{q} d x-\frac{\left\|u_{n}\right\|_{H^{1}}^{p-2}}{p} \int_{\Omega} b(x)\left|\widehat{u}_{n}\right|^{p} d x=o_{n}(1), \\
\left\|\widehat{u}_{n}\right\|_{H^{1}}^{2}-\lambda\left\|u_{n}\right\|_{H^{1}}^{q-2} \int_{\Omega} a(x)\left|\widehat{u}_{n}\right|^{q} d x-\left\|u_{n}\right\|_{H^{1}}^{p-2} \int_{\Omega} b(x)\left|\widehat{u}_{n}\right|^{p} d x=o_{n}(1) .
\end{gathered}
$$

From (4.1)-(4.3), we can deduce that

$$
\left\|\widehat{u}_{n}\right\|_{H^{1}}^{2}=\frac{2(p-q)}{q(p-2)}\left\|u_{n}\right\|^{q-2} \lambda \int_{\Omega} a(x)|\widehat{u}|^{q} d x+o_{n}(1) .
$$


Since $1<q<2$ and $\left\|u_{n}\right\|_{H^{1}} \rightarrow \infty$, (4.4) implies

$$
\left\|\widehat{u}_{n}\right\|_{H^{1}}^{2} \longrightarrow 0 \quad \text { as } n \longrightarrow \infty,
$$

which contradicts with the fact that $\left\|\widehat{u}_{n}\right\|_{H^{1}}=1$ for all $n$.

We assume the condition $\left(\Omega_{b}\right)$ holds and recall

$$
S_{p}^{b}(\Omega)=\inf _{u \in H_{0}^{1}(\Omega) \backslash\{0\}} \frac{\|u\|_{H^{1}}^{2}}{\left(\int_{\Omega} b(x)|u|^{p} d x\right)^{2 / p}} .
$$

Lemma 4.2. Assume that (A1), (B1), and $\left(\Omega_{b}\right)$ hold. If $\left\{u_{n}\right\} \subset H_{0}^{1}(\Omega)$ is a $(P S)_{c}$-sequence for $J_{\lambda a, b}$ with $c \in\left(0, \alpha_{0}^{b}(\Omega)\right)$, then there exists a subsequence of $\left\{u_{n}\right\}$ converging weakly to a nonzero solution of $\left(E_{\lambda a, b}\right)$.

Proof. Let $\left\{u_{n}\right\} \subset H_{0}^{1}(\Omega)$ be a $(P S)_{c}$-sequence for $J_{\lambda a, b}$ with $c \in\left(0, \alpha_{0}^{b}(\Omega)\right)$. We know from Lemma 4.1 that $\left\{u_{n}\right\}$ is bounded in $H_{0}^{1}(\Omega)$, and then there exist a subsequence of $\left\{u_{n}\right\}$ (still denoted by $\left.\left\{u_{n}\right\}\right)$ and $u_{0} \in H_{0}^{1}(\Omega)$ such that

$$
\begin{aligned}
& u_{n} \rightarrow u_{0} \quad \text { weakly in } H_{0}^{1}(\Omega), \\
& u_{n} \longrightarrow u_{0} \quad \text { almost every where in } \Omega, \\
& u_{n} \longrightarrow u_{0} \quad \text { strongly in } L_{\text {loc }}^{s}(\Omega) \forall 1 \leq s<2^{*} .
\end{aligned}
$$

It is easy to see that $\left(J_{\lfloor a, b}\right)^{\prime}\left(u_{0}\right)=0$, and by (A1), Egorov theorem, and Hölder inequality, we have

$$
\lambda \int_{\Omega} a(x)\left|u_{n}\right|^{q} d x=\lambda \int_{\Omega} a(x)\left|u_{0}\right|^{q} d x+o_{n}(1)
$$

Next we verify that $u_{0} \not \equiv 0$. Arguing by contradiction, we assume $u_{0} \equiv 0$. Setting

$$
l=\lim _{n \rightarrow \infty} \int_{\Omega} b(x)\left|u_{n}\right|^{p} d x
$$

Since $\left(J_{\lambda a, b}\right)^{\prime}\left(u_{n}\right)=o_{n}(1)$ and $\left\{u_{n}\right\}$ is bounded, then by (4.8), we can deduce that

$$
0=\lim _{n \rightarrow \infty}\left\langle\left(J_{\lambda a, b}\right)^{\prime}\left(u_{n}\right), u_{n}\right\rangle=\lim _{n \rightarrow \infty}\left(\left\|u_{n}\right\|_{H^{1}}^{2}-\int_{\Omega} b(x)\left|u_{n}\right|^{p} d x\right)=\lim _{n \rightarrow \infty}\left\|u_{n}\right\|_{H^{1}}^{2}-l,
$$


that is,

$$
\lim _{n \rightarrow \infty}\left\|u_{n}\right\|_{H^{1}}^{2}=l
$$

If $l=0$, then we get $c=\lim _{n \rightarrow \infty} J_{\lambda a, b}\left(u_{n}\right)=0$, which contradicts with $c>0$. Thus we conclude that $l>0$. Furthermore, by the definition of $S_{p}^{b}(\Omega)$ we obtain

$$
\left\|u_{n}\right\|_{H^{1}}^{2} \geq S_{p}^{b}(\Omega)\left(\int_{\Omega} b(x)\left|u_{n}\right|^{p} d x\right)^{2 / p}
$$

Then as $n \rightarrow \infty$ we have

$$
l=\lim _{n \rightarrow \infty}\left\|u_{n}\right\|_{H^{1}}^{2} \geq S_{p}^{b}(\Omega) l^{2 / p},
$$

which implies that

$$
1 \geq S_{p}^{b}(\Omega)^{p /(p-2)}
$$

Hence, from (1.7) and (4.8)-(4.14) we get,

$$
\begin{aligned}
c & =\lim _{n \rightarrow \infty} J_{\lambda a, b}\left(u_{n}\right)=\frac{1}{2} \lim _{n \rightarrow \infty}\left\|u_{n}\right\|_{H^{1}}^{2}-\frac{\lambda}{q} \lim _{n \rightarrow \infty} \int_{\Omega} a(x)\left|u_{n}\right|^{q} d x-\frac{1}{p} \lim _{n \rightarrow \infty} \int_{\Omega} b(x)\left|u_{n}\right|^{p} d x \\
& =\left(\frac{1}{2}-\frac{1}{p}\right) l \geq \frac{p-2}{2 p} S_{p}^{b}(\Omega)^{p /(p-2)}=\alpha_{0}^{b}(\Omega) .
\end{aligned}
$$

This is a contradiction to $c<\alpha_{0}^{b}(\Omega)$. Therefore $u_{0}$ is a nonzero solution of $\left(E_{\curlywedge a, b}\right)$.

Lemma 4.3. Assume that (A1)-(A2), (B1), and $\left(\Omega_{b}\right)$ hold. Let $w_{0}$ be a positive ground state solution of $\left(E_{b}\right)$; then
(i) $\sup _{t \geq 0} J_{\lambda a, b}\left(t w_{0}\right)<\alpha_{0}^{b}(\Omega)$ for all $\lambda>0$;
(ii) $\alpha_{\lambda a, b}^{-}<\alpha_{0}^{b}(\Omega)$ for all $\lambda \in\left(0, \Lambda_{0}\right)$.

Proof. (i) First, we consider the functional $Q: H_{0}^{1}(\Omega) \rightarrow \mathbb{R}$ defined by

$$
Q(u)=\frac{1}{2}\|u\|_{H^{1}}^{2}-\frac{1}{p} \int_{\Omega} b(x)|u|^{p} d x \quad \forall u \in H_{0}^{1}(\Omega)
$$


Then, from (1.3) and (1.7), we conclude that

$$
\sup _{t \geq 0} Q\left(t w_{0}\right)=\frac{p-2}{2 p}\left(\frac{\left\|w_{0}\right\|_{H^{1}}^{2}}{\left(\int_{\Omega} b(x)\left|w_{0}\right|^{p} d x\right)^{2 / p}}\right)^{p /(p-2)}=\frac{p-2}{2 p} S_{p}^{b}(\Omega)^{p /(p-2)}=\alpha_{0}^{b}(\Omega)
$$

where the following fact has been used:

$$
\sup _{t \geq 0}\left(\frac{t^{2}}{2} A-\frac{t^{p}}{p} B\right)=\frac{p-2}{2 p}\left(\frac{A}{B^{2 / p}}\right)^{p /(p-2)} \quad \text { where } A, B>0
$$

Using the definitions of $J_{\lambda a, b}, w_{0}$ and $b(x)>0$ for all $x \in \Omega$, for any $\lambda>0$, we have

$$
J_{\lambda a, b}\left(t w_{0}\right) \longrightarrow-\infty \quad \text { as } t \longrightarrow \infty
$$

From this we know that there exists $t_{0}>0$ such that

$$
\sup _{t \geq 0} J_{\lambda a, b}\left(t w_{0}\right)=\sup _{0 \leq t \leq t_{0}} J_{\lambda a, b}\left(t w_{0}\right)
$$

By the continuity of $J_{\lambda a, b}\left(t w_{0}\right)$ as a function of $t \geq 0$ and $J_{\lambda a, b}(0)=0$, we can find some $t_{1} \in\left(0, t_{0}\right)$ such that

$$
\sup _{0 \leq t \leq t_{1}} J_{\lambda a, b}\left(t w_{0}\right)<\alpha_{0}^{b}(\Omega) .
$$

Thus, we only need to show that

$$
\sup _{t_{1} \leq t \leq t_{0}} J_{\lambda a, b}\left(t w_{0}\right)<\alpha_{0}^{b}(\Omega)
$$

To this end, by (A2) and (4.17), we have

$$
\sup _{t_{1} \leq t \leq t_{0}} J_{\lambda a, b}\left(t w_{0}\right) \leq \sup _{t \geq 0} Q\left(t w_{0}\right)-\frac{t_{1}^{q}}{q} \int_{\Omega} a(x)\left|w_{0}\right|^{q} d x<\alpha_{0}^{b}(\Omega) .
$$

Hence (i) holds.

(ii) By (A1), (A2), and the definition of $w_{0}$, we have

$$
\int_{\Omega} b(x)\left|w_{0}\right|^{p} d x>0, \quad \int_{\Omega} a(x)\left|w_{0}\right|^{q} d x>0 .
$$


Combining this with lemma 2.5(ii), from the definition of $\alpha_{\lambda a, b}^{-}$and part (i), for all $\lambda \in\left(0, \Lambda_{0}\right)$, we obtain that there exists $t_{0}>0$ such that $t_{0} w_{0} \in \mathcal{M}_{\lambda a, \mathrm{~b}}^{-}(\Omega)$ and

$$
\alpha_{\lambda a, b}^{-} \leq J_{\lambda a, b}\left(t_{0} w_{0}\right) \leq \sup _{t \geq 0} J_{\lambda a, b}\left(t w_{0}\right)<\alpha_{0}^{b}(\Omega)
$$

Therefore, (ii) holds.

Now, we establish the existence of a local minimum of $J_{\lambda}$ on $\mathcal{M}_{\lambda a, b}^{-}(\Omega)$.

Theorem 4.4. Assume that (A1)-(A2), (B1), and $\left(\Omega_{b}\right)$ hold. If $\lambda \in\left(0,(q / 2) \Lambda_{0}\right)$, then $J_{\lambda a, b}$ has a minimizer $U_{\lambda}$ in $\mathcal{M}_{\lambda a, b}^{-}(\Omega)$ and it satisfies the following.

(i) $J_{\lambda a, b}\left(U_{\curlywedge}\right)=\alpha_{\lambda a, b}^{-}$.

(ii) $U_{\lambda}$ is a positive solution of $\left(E_{\lambda a, b}\right)$ in $\Omega$.

Proof. If $\lambda \in\left(0,(q / 2) \Lambda_{0}\right)$, then by Theorem 3.1(ii), Proposition 3.3(ii), and Lemma 4.3(ii), there exists a $(P S)_{\alpha_{\lambda a, b}^{-}}$-sequence $\left\{u_{n}\right\} \subset \mathcal{M}_{\lambda a, b}^{-}(\Omega)$ in $H_{0}^{1}(\Omega)$ for $J_{\lambda a, b}$ with $\alpha_{\lambda a, b}^{-} \in\left(0, \alpha_{0}^{b}(\Omega)\right)$. From Lemma 4.2, there exist a subsequence still denoted by $\left\{u_{n}\right\}$ and a nonzero solution $U_{\lambda} \in H_{0}^{1}(\Omega)$ of $\left(E_{\lambda a, b}\right)$ such that $u_{n} \rightarrow U_{\lambda}$ weakly in $H_{0}^{1}(\Omega)$. Now we prove that $u_{n} \rightarrow U_{\lambda}$ strongly in $H_{0}^{1}(\Omega)$ and $J_{\curlywedge a, b}\left(U_{\curlywedge}\right)=\alpha_{\lambda a, b}^{-}$. By (3.15), if $u \in \mathcal{M}_{\curlywedge a, b}(\Omega)$, then

$$
J_{\lambda a, b}(u)=\frac{p-2}{2 p}\|u\|_{H^{1}}^{2}-\frac{p-q}{p q} \lambda \int_{\Omega} a(x)|u|^{q} d x .
$$

First, we prove that $U_{\lambda} \in \mathcal{M}_{\lambda a, b}^{-}(\Omega)$. On the contrary, if $U_{\lambda} \in \mathcal{M}_{\lambda a, b}^{+}(\Omega)$, then by $\mathcal{M}_{\lambda a, b}^{-}(\Omega)$ being closed in $H_{0}^{1}(\Omega)$, we have $\left\|U_{\lambda}\right\|_{H^{1}}^{2}<\liminf _{n \rightarrow \infty}\left\|u_{n}\right\|_{H^{1}}^{2}$. From Lemma 2.3(i) and $b(x)>0$ for all $x \in \Omega$, we get

$$
\int_{\Omega} a(x)\left|U_{\lambda}\right|^{q} d x>0, \quad \int_{\Omega} b(x)\left|U_{\lambda}\right|^{p} d x>0
$$

By Lemma 2.5(ii), there exists a unique $t_{\lambda}^{-}$such that $t_{\lambda}^{-} U_{\lambda} \in \mathcal{M}_{\lambda a, b}^{-}(\Omega)$. Since $u_{n} \in \mathcal{M}_{\lambda a, b}^{-}(\Omega)$, $J_{\lambda a, b}\left(u_{n}\right) \geq J_{\curlywedge a, b}\left(t u_{n}\right)$ for all $t \geq 0$ and by (4.26), we have

$$
\alpha_{\lambda a, b}^{-} \leq J_{\lambda a, b}\left(t_{\lambda}^{-} U_{\curlywedge}\right)<\lim _{n \rightarrow \infty} J_{\lambda a, b}\left(t_{\lambda}^{-} u_{n}\right) \leq \lim _{n \rightarrow \infty} J_{\lambda a, b}\left(u_{n}\right)=\alpha_{\lambda a, b}^{-}
$$

and this is a contradiction. In order to prove that $J_{\lambda a, b}\left(U_{\curlywedge}\right)=\alpha_{\lambda a, b}^{-}$, it suffices to recall that $u_{n}$ $U_{\lambda} \in \mathcal{M}_{\lambda a, b}^{-}$for all $n$, by (4.26) and applying Fatou's lemma to get

$$
\begin{aligned}
\alpha_{\lambda a, b}^{-} & \leq J_{\lambda a, b}\left(U_{\curlywedge}\right)=\frac{p-2}{2 p}\left\|U_{\curlywedge}\right\|_{H^{1}}^{2}-\frac{p-q}{p q} \lambda \int_{\Omega} a(x)\left|U_{\curlywedge}\right|^{q} d x \\
& \leq \liminf _{n \rightarrow \infty}\left(\frac{p-2}{2 p}\left\|u_{n}\right\|_{H^{1}}^{2}-\frac{p-q}{p q} \lambda \int_{\Omega} a(x)\left|u_{n}\right|^{q} d x\right) \\
& \leq \liminf _{n \rightarrow \infty} J_{\lambda a, b}\left(u_{n}\right)=\alpha_{\lambda a, b}^{-} .
\end{aligned}
$$


This implies that $J_{\lambda a, b}\left(U_{\lambda}\right)=\alpha_{\lambda a, b}^{-}$and $\lim _{n \rightarrow \infty}\left\|u_{n}\right\|_{H^{1}}^{2}=\left\|U_{\lambda}\right\|_{H^{1}}^{2}$. Let $v_{n}=u_{n}-U_{\lambda}$; then by Brézis and Lieb, lemma [33] implies that

$$
\left\|v_{n}\right\|_{H^{1}}^{2}=\left\|u_{n}\right\|_{H^{1}}^{2}-\left\|U_{\lambda}\right\|_{H^{1}}^{2}+o_{n}(1)
$$

Therefore, $u_{n} \rightarrow U_{\curlywedge}$ strongly in $H_{0}^{1}(\Omega)$.

Since $J_{\lambda a, b}\left(U_{\lambda}\right)=J_{\lambda a, b}\left(\left|U_{\lambda}\right|\right)$ and $\left|U_{\lambda}\right| \in \mathcal{M}_{\lambda a, b}^{-}(\Omega)$, by Lemma 2.2 we may assume that $U_{\lambda}$ is a nonzero nonnegative solution of $\left(E_{\lambda a, b}\right)$. Finally, By the Harnack inequality [34] we deduce that $U_{\lambda}>0$ in $\Omega$.

Now, we complete the proof of Theorem 1.2: by Theorems 3.4, 4.4, we obtain that $\left(E_{\lambda a, b}\right)$ has two positive solutions $u_{\lambda}$ and $U_{\lambda}$ such that $u_{\lambda} \in \mathcal{M}_{\lambda a, b}^{+}(\Omega), U_{\lambda} \in \mathcal{M}_{\lambda a, b}^{-}(\Omega)$. Since $\mathcal{M}_{\lambda a, b}^{+}(\Omega) \cap \mathcal{M}_{\lambda a, b}^{-}(\Omega)=\emptyset$, this implies that $u_{\curlywedge}$ and $U_{\curlywedge}$ are distinct.

\section{References}

[1] A. Ambrosetti, H. Brézis, and G. Cerami, "Combined effects of concave and convex nonlinearities in some elliptic problems," Journal of Functional Analysis, vol. 122, no. 2, pp. 519-543, 1994.

[2] Adimurthi, F. Pacella, and S. L. Yadava, "On the number of positive solutions of some semilinear Dirichlet problems in a ball," Differential and Integral Equations, vol. 10, no. 6, pp. 1157-1170, 1997.

[3] L. Damascelli, M. Grossi, and F. Pacella, "Qualitative properties of positive solutions of semilinear elliptic equations in symmetric domains via the maximum principle," Annales de l'Institut Henri Poincaré. Analyse Non Linéaire, vol. 16, no. 5, pp. 631-652, 1999.

[4] T. Ouyang and J. Shi, "Exact multiplicity of positive solutions for a class of semilinear problem. II," Journal of Differential Equations, vol. 158, no. 1, pp. 94-151, 1999.

[5] M. Tang, "Exact multiplicity for semilinear elliptic Dirichlet problems involving concave and convex nonlinearities," Proceedings of the Royal Society of Edinburgh. Section A, vol. 133, no. 3, pp. 705-717, 2003.

[6] A. Ambrosetti, J. Garcia Azorero, and I. Peral, "Multiplicity results for some nonlinear elliptic equations," Journal of Functional Analysis, vol. 137, no. 1, pp. 219-242, 1996.

[7] J. P. García Azorero, I. Peral Alonso, and J. J. Manfredi, "Sobolev versus Hölder local minimizers and global multiplicity for some quasilinear elliptic equations," Communications in Contemporary Mathematics, vol. 2, no. 3, pp. 385-404, 2000.

[8] K. J. Brown and T.-F. Wu, "A fibering map approach to a semilinear elliptic boundary value problem," Electronic Journal of Differential Equations, vol. 2007, no. 69, pp. 1-9, 2007.

[9] K. J. Brown and Y. Zhang, "The Nehari manifold for a semilinear elliptic equation with a signchanging weight function," Journal of Differential Equations, vol. 193, no. 2, pp. 481-499, 2003.

[10] D. Cao and X. Zhong, "Multiplicity of positive solutions for semilinear elliptic equations involving the critical Sobolev exponents," Nonlinear Analysis: Theory, Methods E Applications, vol. 29, no. 4, pp. 461-483, 1997.

[11] D. G. de Figueiredo, J.-P. Gossez, and P. Ubilla, "Multiplicity results for a family of semilinear elliptic problems under local superlinearity and sublinearity," Journal of the European Mathematical Society, vol. 8, no. 2, pp. 269-286, 2006.

[12] J. Chabrowski and J. M. Bezzera do Ó, "On semilinear elliptic equations involving concave and convex nonlinearities," Mathematische Nachrichten, vol. 233-234, pp. 55-76, 2002.

[13] J. V. Gonçalves and O. H. Miyagaki, "Multiple positive solutions for semilinear elliptic equations in $\mathbb{R}^{N}$ involving subcritical exponents," Nonlinear Analysis: Theory, Methods $\mathcal{E}$ Applications, vol. 32, no. 1, pp. 41-51, 1998.

[14] Z. Liu and Z.-Q. Wang, "Schrödinger equations with concave and convex nonlinearities," Zeitschrift für Angewandte Mathematik und Physik, vol. 56, no. 4, pp. 609-629, 2005.

[15] T.-F. Wu, "Multiplicity of positive solutions for semilinear elliptic equations in $\mathbb{R}^{N}$," Proceedings of the Royal Society of Edinburgh. Section A, vol. 138, no. 3, pp. 647-670, 2008.

[16] T.-F. Wu, "Multiple positive solutions for a class of concave-convex elliptic problems in $\mathbb{R}^{N}$ involving sign-changing weight," Journal of Functional Analysis, vol. 258, no. 1, pp. 99-131, 2010. 
[17] T.-F. Wu, "Multiple positive solutions for Dirichlet problems involving concave and convex nonlinearities," Nonlinear Analysis: Theory, Methods E Applications, vol. 69, no. 12, pp. 4301-4323, 2008.

[18] M. L. Miotto and O. H. Miyagaki, "Multiple positive solutions for semilinear Dirichlet problems with sign-changing weight function in infinite strip domains," Nonlinear Analysis: Theory, Methods E Applications, vol. 71, no. 7-8, pp. 3434-3447, 2009.

[19] T.-S. Hsu and H.-L. Lin, "Multiple positive solutions for semilinear elliptic equations in $\mathbb{R}^{N}$ involving concave-convex nonlinearities and sign-changing weight functions," Abstract and Applied Analysis, vol. 2010, Article ID 658397, 21 pages, 2010.

[20] G. Tarantello, "On nonhomogeneous elliptic equations involving critical Sobolev exponent," Annales de l'Institut Henri Poincaré. Analyse Non Linéaire, vol. 9, no. 3, pp. 281-304, 1992.

[21] I. Ekeland, "On the variational principle," Journal of Mathematical Analysis and Applications, vol. 47, pp. 324-353, 1974.

[22] P. H. Rabinowitz, Minimax Methods in Critical Point Theory with Applications to Differential Equations, vol. 65 of CBMS Regional Conference Series in Mathematics, American Mathematical Society, Washington, DC, USA, 1986.

[23] A. Ambrosetti and P. H. Rabinowitz, "Dual variational methods in critical point theory and applications," Journal of Functional Analysis, vol. 14, pp. 349-381, 1973.

[24] M. Willem, Minimax Theorems, vol. 24 of Progress in Nonlinear Differential Equations and Their Applications, Birkhäuser, Boston, Mass, USA, 1996.

[25] H. Berestycki and P.-L. Lions, "Nonlinear scalar field equations. I. Existence of a ground state," Archive for Rational Mechanics and Analysis, vol. 82, no. 4, pp. 313-345, 1983.

[26] W. C. Lien, S. Y. Tzeng, and H. C. Wang, "Existence of solutions of semilinear elliptic problems on unbounded domains," Differential and Integral Equations, vol. 6, no. 6, pp. 1281-1298, 1993.

[27] K.-J. Chen and H.-C. Wang, "A necessary and sufficient condition for Palais-Smale conditions," SIAM Journal on Mathematical Analysis, vol. 31, no. 1, pp. 154-165, 1999.

[28] M. del Pino and P. L. Felmer, "Local mountain passes for semilinear elliptic problems in unbounded domains," Calculus of Variations and Partial Differential Equations, vol. 4, no. 2, pp. 121-137, 1996.

[29] M. A. del Pino and P. L. Felmer, "Least energy solutions for elliptic equations in unbounded domains," Proceedings of the Royal Society of Edinburgh. Section A, vol. 126, no. 1, pp. 195-208, 1996.

[30] M. K. Kwong, "Uniqueness of positive solutions of $\Delta u-u+u^{p}=0$ in $\mathbb{R}^{N}$," Archive for Rational Mechanics and Analysis, vol. 105, no. 3, pp. 243-266, 1989.

[31] P. A. Binding, P. Drábek, and Y. X. Huang, "On Neumann boundary value problems for some quasilinear elliptic equations," Electronic Journal of Differential Equations, vol. 1997, no. 5, pp. 1-11, 1997.

[32] T.-F. Wu, "On semilinear elliptic equations involving concave-convex nonlinearities and signchanging weight function," Journal of Mathematical Analysis and Applications, vol. 318, no. 1, pp. 253$270,2006$.

[33] H. Brézis and E. Lieb, "A relation between pointwise convergence of functions and convergence of functionals," Proceedings of the American Mathematical Society, vol. 88, no. 3, pp. 486-490, 1983.

[34] N.S. Trudinger, "On Harnack type inequalities and their application to quasilinear elliptic equations," Communications on Pure and Applied Mathematics, vol. 20, pp. 721-747, 1967. 